\title{
UNE DOUBLE GÉNÉRALISATION DU THÉORÈME DE FEJÉR-LEBESGUE
}

\author{
PAR \\ SABIN LESSARD
}

\begin{abstract}
SOMMAIRE. Nous nous emploierons dans cet article à démontrer la convergence presque partout pour les moyennes de projections orthogonales croissantes sur $L^{2}$, qui sont ou bien u.a.c. ou bien positives, ce qui nous donnera une double généralisation du théorème classique de Fejér-Lebesgue pour les séries de Fourier. Enfin nous terminerons en énonçant un résultat général qui englobe tous ceux connus jusqu'á maintenant avec la condition u.a.c. (cf. R. Duncan, Can. Math. Bull. 20 (1977) 277-284).
\end{abstract}

Introduction. Dans cet exposé, tous les opérateurs porteront sur l'espace $L^{2}$ des fonctions à valeurs réelles définies sur un espace mesuré $(\Omega, \mathscr{A}, \mu)$ qu'on supposera d'abord fini. En général, nous dirons que la suite d'opérateurs $\left\{T_{k}\right\}_{k \geq 1}$ est uniformément absolument continue (u.a.c.) s'il existe une mesure positive $\nu$ sur $\mathscr{A}$ telle que

$$
\int\left|T_{k} I_{\mathrm{A}}\right| d \mu \leq \nu(A)<\infty
$$

pour tout ensemble $\mu$-fini $A$ et pour tout $k \geq 1$. Cette condition a été introduite pour la première fois par R. Duncan ([1] et [2]). D'autre part, nous dirons que la suite $\left\{T_{k}\right\}_{k \geq 1}$ est positive si

pour tout $k \geq 1$.

$$
T_{k} f \geq 0 \quad \text { p.p. dès que } f \geq 0 \quad \text { p.p. }
$$

R. Duncan a démontré de façon originale que pour une suite croissante de projections orthogonales $\left\{\boldsymbol{P}_{k}\right\}_{k \geq 1}$ sur $L^{2}$ qui est ou bien u.a.c. ou bien positive, on a la convergence p.p. des $P_{k} f$ pour tout $f$ dans $L^{2}$. E. M. Stein ([5], p. 87) avait déjà démontré le résultat sous la condition de positivité, mais en utilisant cependant des arguments bien différents. Nous nous proposons ici d'étudier ce qui se passe pour les moyennes de projections orthogonales croissantes.

Résultat principal. Soit $\left\{P_{j}\right\}_{j \geq 1}$ une suite croissante de projections orthogonales sur $L^{2}$ et $T_{k}=\frac{1}{k} \sum_{j=1}^{k} P_{j}$ pour tout $k \geq 1$. La structure particulière des $T_{k}$ nous permet d'obtenir le lemme fondamental suivant:

LEMME. $T_{k} \circ T_{n}^{*}=\frac{n-k-1}{n} T_{k}+\frac{1}{k} \sum_{r=1}^{k} \frac{2 r}{n} T_{r}$ dès que $1 \leq k \leq n$.

Reçu par les rédacteurs le 15 mai 1978 et, dans sa version revisée, le 3 janvier 1979. 
Démonstration. Par les propriétés même de projections orthogonales croissantes et quelques manipulations techniques, on obtient les égalités successives suivantes:

$$
\begin{aligned}
T_{k} \circ T_{n}^{*} & =\left(\frac{1}{k} \sum_{j=1}^{k} P_{j}\right) \circ\left(\frac{1}{n} \sum_{l=1}^{n} P_{l}\right) \\
& =\frac{1}{k n} \sum_{j=1}^{k} \sum_{l=1}^{n} P \min (j, l) \\
& =\frac{1}{k n} \sum_{m=1}^{k}(n+k-2 m+1) P_{m} \\
& =\frac{1}{k n} \sum_{m=1}^{k}(n-k-1) P_{m}+\frac{1}{k n} \sum_{m=1}^{k} 2(k-m+1) P_{m} \\
& =\frac{n-k-1}{n}\left(\frac{1}{k} \sum_{m=1}^{k} P_{m}\right)+\frac{1}{k} \sum_{r=1}^{k} \frac{2}{n}\left(\sum_{m=1}^{r} P_{m}\right) \\
& =\frac{n-k-1}{n} T_{k}+\frac{1}{k} \sum_{r=1}^{k} \frac{2 r}{n} T_{r} \square
\end{aligned}
$$

Maintenant, en utilisant les techniques développées par R. Duncan, on peut démontrer une inégalité du maximum sous l'hypothèse u.a.c.

THÉORÈME 1. Si la suite $\left\{T_{k}\right\}_{k \geq 1}$ est u.a.c., alors $\left\|\sup _{k}\left|T_{k} f\right|\right\|_{1} \leq 2 \sqrt{6 \nu(\Omega)}\|f\|_{2}$ pour tout $f$ dans $L^{2}$.

DÉmonstration. Il suffit de vérifier le critère donné par $\mathrm{R}$. Duncan, à savoir que pour n'importe quelle famille finie $\left\{B_{k}: k=1, \ldots, N\right\}$ d'ensembles mesurables disjoints, on a

$$
\left\|\sum_{k=1}^{N} T_{k}^{*} I_{B_{k}}\right\|_{2} \leq \sqrt{6 \nu(\Omega)} .
$$

En effet

$$
\begin{aligned}
\left\|\sum_{k=1}^{N} T_{k}^{*} I_{B_{k}}\right\|_{2}^{2}=\sum_{n=1}^{N} \sum_{k=1}^{N} \int T_{n}^{*} I_{B_{n}} T_{k}^{*} I_{B_{k}} & \\
& =\sum_{n=1}^{N} \sum_{k=1}^{n} \int_{B_{k}} T_{k} \circ T_{n}^{*} I_{B_{n}}+\sum_{n=2}^{N} \sum_{k=1}^{n-1} \int_{B_{k}} T_{k} \circ T_{n}^{*} I_{B_{n}}
\end{aligned}
$$

Mais chacune de ces sommes peut se décomposer en deux parties par le lemme précédent. Prenons par exemple la première et notons par (I) et (II) les deux 
parties de sa décomposition. On obtient alors les majorations suivantes:

$$
\begin{aligned}
\text { II } & =\sum_{n=1}^{N} \sum_{k=1}^{n} \frac{n-k-1}{n} \int_{B_{k}} T_{k} I_{B_{n}} \\
& =\sum_{k=1}^{N} \sum_{n=k}^{N} \frac{n-k-1}{n} \int_{B_{n}} T_{k}^{*} I_{B_{k}} \\
& \leq \sum_{k=1}^{N} \sum_{n=k}^{N} \int_{B_{n}}\left|T_{k} I_{B_{k}}\right| \\
& \leq \sum_{k=1}^{N} \int\left|T_{k} I_{B_{k}}\right| \\
& \leq \sum_{k=1}^{N} \nu\left(B_{k}\right) \\
& \leq \nu(\Omega) \\
\text { III } & =\sum_{n=1}^{N} \sum_{k=1}^{n} \frac{1}{k} \sum_{r=1}^{k} \frac{2 r}{n} \int_{B_{k}} T_{r} I_{B_{n}} \\
& =\sum_{k=1}^{N} \frac{1}{k} \sum_{r=1}^{k} \sum_{n=k}^{N} \frac{2 r}{n} \int_{B_{n}} T_{r}^{*} I_{B_{k}} \\
& \leq \sum_{k=1}^{N} \frac{1}{k} \sum_{r=1}^{k} \sum_{n=k}^{N} 2 \int_{B_{n}}\left|T_{r} I_{B_{k}}\right| \\
& \leq 2 \sum_{k=1}^{N} \frac{1}{k} \sum_{r=1}^{k} \int\left|T_{r} I_{B_{k}}\right| \\
& \leq 2 \sum_{k=1}^{N} \nu\left(B_{k}\right) \\
& \leq 2 \nu(\Omega)
\end{aligned}
$$

Pour la deuxième somme, on obtient évidemment les mêmes majorations qui, rassemblées ensemble, nous donnent bien le résultat annoncé.

Le lemme précédent va nous permettre d'obtenir aussi une inégalité du maximum dans le cas positif. Cependant nous allons suivre cette fois-ci les idées de E. M. Stein lorsqu'il a démontré une inégalité du maximum dans le cas des moyennes des itérés d'un opérateur positif auto-adjoint contractant sur $L^{2}$.

ThÉORÈME 2. Si la suite $\left\{T_{k}\right\}_{k \geq 1}$ est positive, alors $\left\|\sup _{k}\left|T_{k} f\right|\right\|_{2} \leq 6\|f\|_{2}$ pour tout $f$ dans $L^{2}$.

Démonstration. Remarquons d'abord qu'il suffit de démontrer l'inégalité $\left\|\sup _{k}\left|(1-\delta)^{k} T_{k} f\right|\right\|_{2} \leq 6\|f\|_{2}$ pour $0<\delta<1$ arbitraire car pour n'importe quel 
$N \geq 1$

$$
\left\|\max _{1 \leq k \leq N}\left|T_{k} f\right|\right\|_{2} \leq \frac{1}{(1-\delta)^{N}}\left\|\max _{1 \leq k \leq N}\left|(1-\delta)^{k} T_{k} f\right|\right\|_{2}
$$

Si on définit $U_{k}=(1-\delta)^{k} T_{k}$, alors pour tout $f$ positif, dans le cas où $1 \leq k \leq n$

$$
\begin{aligned}
U_{k} \circ U_{n} f & =(1-\delta)^{k+n}\left[\frac{n-k-1}{n} T_{k} f+\frac{1}{k} \sum_{r=1}^{k} \frac{2 r}{n} T_{r} f\right] \\
& \leq U_{k} f+\frac{2}{k} \sum_{r=1}^{k} U_{r} f
\end{aligned}
$$

et donc dans tous les cas

$$
U_{k} \circ U_{n} f \leq U_{k} f+\frac{2}{k} \sum_{r=1}^{k} U_{r} f+U_{n} f+\frac{2}{n} \sum_{r=1}^{n} U_{r} f
$$

D'autre part pour tout $f$ dans $L^{2}$

$$
\int\left(\sup _{k}\left|U_{k} f\right|\right)^{2} \leq \sum_{k=1}^{\infty} \int\left(U_{k} f\right)^{2} \leq C_{\delta}^{2} \int f^{2}
$$

où $C_{\delta}^{2}=\sum_{k=1}^{\infty}(1-\delta)^{2 k}<\infty$. Soit alors $C$ la plus petite constante telle que $\left\|\sup _{k}\left|U_{k} f\right|\right\|_{2} \leq C\|f\|_{2}$ pour tout $f$ dans $L^{2}$. On va montrer que $C \leq 6$.

Soit $0<\varepsilon<1$ arbitraire. Par la définition même de $C$, il existe nécessairement une fonction mesurable $n(x)$ définie sur $\Omega$ à valeurs entières telle que l'opérateur $B$ sur $L^{2}$ donné par $B f(x)=U_{n(x)} f(x)$ vérifie $\|B\|_{2} \geq$ $C(1-\varepsilon)$. On définit aussi l'opérateur $B_{1}$ sur $L^{2}$ par $B_{1} f(x)=\frac{1}{n(x)} \sum_{r=1}^{n(x)} U_{r} f(x)$. Alors les opérateurs $B$ et $B_{1}$ sont positifs, et pour tout $f$ positif, on trouve successivement

$$
B \circ U_{n} f(x) \leq B f(x)+2 B_{1} f(x)+U_{n} f(x)+\frac{2}{n} \sum_{r=1}^{n} U_{r} f(x)
$$

puis

$$
B \circ B^{*} f(x) \leq B^{*} f(x)+2 B_{1}^{*} f(x)+B f(x)+2 B_{1} f(x)
$$

On obtient donc finalement les inégalités

$$
C^{2}(1-\varepsilon)^{2} \leq\left\|B B^{*}\right\|_{2} \leq\left\|B^{*}+2 B_{1}^{*}+B+2 B_{1}\right\|_{2} \leq 6 C
$$

Ceci n'est possible que si $C \leq 6$. 
Comme on sait que les $\boldsymbol{P}_{j}$ convergent dans la topologie forte des opérateurs sur $L^{2}$ vers une projection orthogonale $P$, il est facile de trouver un sous ensemble dense dans $L^{2}$ pour lequel on a la convergence p.p., en l'occurrence $\left\{(I-P) f: f \in L^{2}\right\} \oplus \bigcup_{N \geq 1}\left\{P_{N} f: f \in L^{2}\right\}$, et alors le principe de Banach ([3], p. 332) nous donne le corollaire suivant à la suite des théorèmes 1 et 2 :

RÉSUltAT PRINCIPAL. Si la suite $\left\{T_{k}\right\}_{k \leq 1}$ est u.a.c. ou positive, alors la suite $\left\{T_{k} f\right\}_{k \leq 1}$ converge p.p. pour tout $f$ dans $L^{2}$.

Comme application, prenons en particulier les $P_{j}$ donnés par les sommes partielles des séries de Fourier. On a alors la représentation intégrale suivante pour les $T_{k}$ :

où

$$
T_{k} f(x)=\frac{1}{2 \pi} \int_{-\pi}^{\pi} F_{k}(t-x) f(t) d t
$$

$$
F_{k}(u)=\frac{1}{k}\left(\frac{\sin k \frac{u}{2}}{\sin \frac{u}{2}}\right)^{2}
$$

est non seulement positif, mais posséde aussi la propriété

$$
\frac{1}{2 \pi} \int_{-\pi}^{\pi} F_{k}(t-x) d x=1 .
$$

Par conséquent la suite $\left\{T_{k}\right\}_{k \geq 1}$ est non seulement positive, mais possède aussi la propriété u.a.c. avec pour $\nu$ la mesure de Lebesgue sur $[-\pi, \pi]$. On voit donc dans ce cas qu'il y a en fait deux fois trop de raisons pour avoir la convergence p.p. des $T_{k} f$ pour tout $f$ dans $L^{2}[-\pi, \pi]$, théorème connu comme celui de Fejér-Lebesgue ([6], p. 90). Pour les $P_{j}$ eux-mêmes, on ne peut malheureusement rien dire car le noyau de leur représentation intégrale ne possède aucune des bonnes propriétés des $\boldsymbol{F}_{\boldsymbol{k}}$. Dans le cas plus général où les $\boldsymbol{P}_{\boldsymbol{j}}$ sont donnés par les sommes partielles de séries orthogonales engendrées par un système de fonctions orthonormées $\left\{\psi_{r}\right\}_{r \geq 1}$ dans $L^{2}$, on trouve pour $T_{k}$ la représentation

$$
T_{k} f=\int F_{k}(x, t) f(t) d \mu(t)
$$

où

$$
F_{k}(x, t)=\sum_{r=1}^{k}\left(1-\frac{r-1}{k}\right) \psi_{r}(x) \psi_{r}(t)
$$

On aura donc la convergence p.p. si le

$$
\sup _{k} \int\left|F_{k}(x, t)\right| d \mu(x)
$$


est intégrable, résultat déjà obtenu par Kaczmarz ([4], p. 109), ou encore simplement si le noyau $F_{k}$ est positif.

Extensions. On admet maintenant la possibilité que la mesure $\mu$ puisse être infinie et on considère une suite quelconque d'opérateurs $\left\{T_{k}\right\}_{k \geq 1}$ sur $L^{2}$ ayant la propriété que pour $1 \leq k \leq n$

$$
T_{k} \circ T_{n}^{*}=\alpha(k, n) Q_{k}+\beta(k, n) Q_{n}^{*}+\frac{1}{k} \sum_{r=1}^{m k} \gamma_{r}(k, n) Q_{r}+\frac{1}{n} \sum_{r=1}^{m n} \delta_{r}(k, n) Q_{r}^{*}
$$

où $\left\{Q_{r}^{*}\right\}_{r \geq 1}$ est une suite d'opérateurs sur $L^{2}$ u.a.c., $m$ est un entier positif et les $\alpha(k, n), \beta(k, n), \gamma_{r}(k, n), \delta_{r}(k, n)$ sont des réels bornés par $M$. On aura alors pour n'importe quel ensemble $\mu$-fini $A$

$$
\int_{A} \sup _{k}\left|T_{k} f\right| \leq 4[(m+1) M \nu(A)]^{1 / 2}\|f\|_{2}
$$

pour tout $f$ dans $L^{2}$. En effet remarquons d'abord que pour n'importe quel $N \geq 1$, on peut écrire

$$
\begin{aligned}
\max _{1 \leq k \leq N}\left|T_{k} f\right| & =\sum_{k=1}^{N} I_{B_{k}}\left|T_{k} f\right| \\
& =\sum_{k=1}^{N} I_{B_{k}}+T_{k} f-\sum_{k=1}^{N} I_{B_{k}}-T_{k} f
\end{aligned}
$$

où les $\boldsymbol{B}_{k}^{+}$(respectivement $B_{k}^{-}$) sont des ensembles mesurables disjoints, et alors

$$
\begin{aligned}
\int_{\mathrm{A}} \max _{1 \leq k \leq N}\left|T_{k} f\right|=\int f \sum_{k=1}^{N} T_{k}^{*} I_{\mathrm{A} \cap \mathrm{B}_{k}^{+}}-\int f & \sum_{k=1}^{N} T_{k}^{*} I_{\mathrm{A} \cap \mathrm{B}_{k}^{-}} \\
& \leq\left(\left\|\sum_{k=1}^{N} T_{k}^{*} I_{\mathrm{A} \cap \mathrm{B}_{k}^{+}}\right\|_{2}+\left\|\sum_{k=1}^{N} T_{k}^{*} I_{\mathrm{A} \cap \mathrm{B}_{k}^{-}}\right\|\right)\|f\|_{2}
\end{aligned}
$$

Les majorations finales peuvent à ce moment-là être obtenues de la même façon que celles obtenues dans la démonstration du théorème 1.

$\mathrm{Si}$ on réussit alors à trouver un sous-ensemble dense dans $L^{2}$ pour lequel on a la convergence p. p., le principe de Banach donnera la convergence p. p. des $I_{\mathrm{A}} T_{k} f$ pour tout $f$ dans $L^{2}$ et tout ensemble $\mu$-fini $A$. Mais pour un $f$ donné, tous les $T_{k} f$ sont nuls en dehors d'un même ensemble de mesure $\sigma$-finie, et par conséquent les $T_{k} f$ convergeront p. p. pour tout $f$ dans $L^{2}$.

Remarquons finalement qu'on peut facilement montrer que tous les opérateurs considérés par R. Duncan avec la condition u.a.c., autant ceux liés à la théorie ergodique que ceux liés à la théorie des martingales, sont dans la classe définie ci-dessus. 
REMERCIEMENTS. Le matériel de cet article fait partie d'une thèse de doctorat présentée récemment à l'Université de Montréal. L'auteur tient à remercier sincèrement Monsieur Richard Duncan qui a agi à titre de directeur de recherche.

\section{RÉFÉRENCES}

1. R. Duncan, Pointwise Convergence Theorems for Self-Adjoint and Unitary Contractions, Ann. of Prob. 5, 622-626 (1977).

2. R. Duncan, Some Pointwise Convergence Results in $L^{P}(\mu), 1<p<\infty$, Can. Math. Bull. 20, 277-284 (1977).

3. N. Dunford and J. T. Schwartz, Linear Operators, Part I, Wiley-Interscience (New York, 1958).

4. S. Kaczmarz, Sur la convergence et la sommabilité des développements orthogonaux, Studia Math. 1, 87-121 (1929).

5. E. M. Stein, Topics in Harmonic Analysis, Ann. Math. Studies 63, Princeton Univ. Press (1970).

6. A. Zygmund, Trigonometric Series, Cambridge Univ. Press, 2nd ed., vol. 1 (New York, 1968).

Centre de Recherche de Mathématiques Appliquées

UnIVERSITÉ dE MonTRÉAL

C.P. 6128 , SuCCurSAle "A"

MONTRÉAL, QUÉBEC H3C $3 J 7$ 